\title{
Erratum to: Preliminary biochemical characterization of the novel, non-AT1, non-AT2 angiotensin binding site from the rat brain
}

\author{
Vardan T. Karamyan • Jason Arsenault • \\ Emanuel Escher • Robert C. Speth
}

Published online: 6 May 2010

(C) Springer Science+Business Media, LLC 2010

\section{Erratum to: Endocr}

DOI 10.1007/s12020-010-9328-2

In the original publication, the right panel of Figure 3 contains an error in the $\mathrm{x}$ axis. The corrected panel appears here.

The online version of the original article can be found under doi: 10.1007/s12020-010-9328-2.

\section{T. Karamyan}

Department of Pharmaceutical Sciences, School of Pharmacy,

Texas Tech University HSC, Amarillo, TX 79106, USA

e-mail: vardan.karamyan@ttuhsc.edu

\section{J. Arsenault · E. Escher}

Department of Pharmacology, Université de Sherbrooke,

Sherbrooke, QC, Canada

e-mail: Jason.Arsenault@USherbrooke.ca

\section{E. Escher}

e-mail: emanuel.escher@USherbrooke.ca

\section{R. C. Speth $(\square)$}

Department of Pharmaceutical Sciences, College of Pharmacy,

Nova Southeastern University, 3200 S. University Dr, Fort

Lauderdale, FL 33328-2018, USA

e-mail: Robert.speth@nova.edu

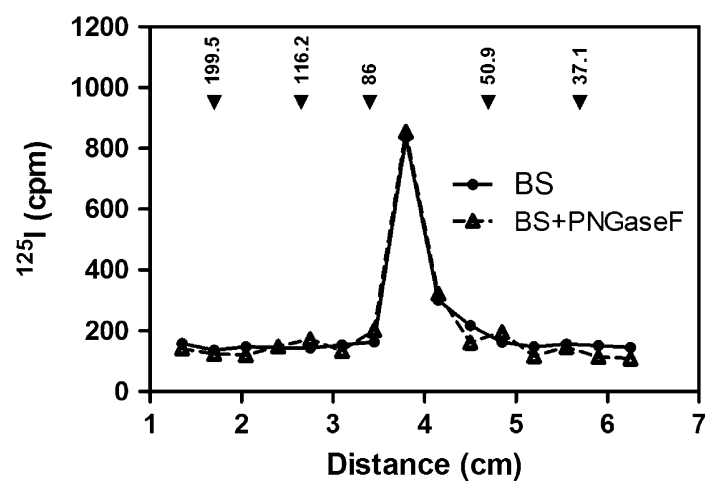

Fig. 3 Analysis of N-glycosylation status of the novel angiotensin binding site (representative results from three independent experiments). Left SDS-PAGE (7.5\%) analysis and Coomassie blue staining of radio-photolabeled ( ${ }^{125} \mathrm{I}-\mathrm{SB}$ pa-AngII), semipurified novel angiotensin binding site without (BS) and with (BS + PNGaseF) PNGaseF treatment. Glycoprotein fetuin was used as a positive control in this experiment. Right migration of ${ }^{125}$ iodine in the "BS" and "BS + PNGase" lanes of the same SDS gel ( $\sim 0.3 \mathrm{~cm}$ sections) 